\title{
O MINI-EXAME DO ESTADO MENTAL (MEM) COMO INDICADOR DE DISFUNÇÃO COGNITIVA APÓS TCE GRAVE
}

\author{
Maria Sumie Koizumi* \\ Regina Márcia Cardoso de Sousa** \\ Mirna Namie Okamura***
}

KOIZUMI, M.S. et al. O Mini-exame do estado mental (MEM) como indicador de disfunção cognitiva apos TCE grave. Rev.Esc.Enf.USP, v.28,n.3, p.281-92, dez. 1994.

O objetivo deste estudo foi analisar as disfunçōes cognitivas apresentadas pelos pacientes que iniciam tratamento ambulatorial após traumatismo crânio-encefálico grave. Durante 1 ano, consecutivamente, 87 pacientes foram atendidos em primeira consulta médica e de enfermagem. Destes, 80 foram submetidos ao Mini-exame do Estado Mental (MEM).

O tempo médio entre o trauma e a aplicação desse exame foi de 74 dias. Utilizou-se como ponto de corte o escore igual ou inferior a 23, obteve-se $35 \%$ de pacientes com disfunção cognitiva. Nos pacientes com $M E M \leq 23$ uma relaçào de 2:1 foi observada quando comparou-se o grupo de pacientes com escolaridade inferior a 4 anos com aquele superior a 4 anos. Os resultados dos testes estatísticos indicam que houve uma forte associação negativa entre a obtenção de pontuação igual ou maior que 24 e a escolaridade maior que 4 anos $(Q=-0,9)$. Ainda nos pacientes com $M E M \leq 23, o$ único item pouco afetado foi o registro de dados. Os demais itens, orientação, atenção e cálculo, memória de fixaçāo e linguagem foram afetados tanto naqueles com escolaridade inferior como superior a 4 anos.

Os resultados obtidos indicam que além das intervençōes de enfermagem decorrentes do diagnóstico disfunção cognitiva dirigidas especificamente ao paciente é preciso estar atento para incluir a familia no tratamento, seja no que se refere ao esclarecimento das condiçōes do paciente, seja na forma de tratá-lo nesta fase de convalescença.

UNITERMOS: Disfunçảo cognitiva. Estado mental. Traumatismo crânio-encefálico.

* Enfermeira.Professor-titular do Departamento de Enfermagem Médico-Cirúrgica da Escola de Enfermagem da USP.

** Enfermeira.Assistente do Departamento de Enfermagem Médico-Cirúrgica da Escola de Enfermagem da USP.

*** Enfermeira da Secretaria Municipal de Saúde de Sảo Paulo. 


\section{INTRODUÇÃO}

As disfunções cognitivas são frequentes após Traumatismo Crânio-Encefálico (TCE) grave. À enfermeira compete também detectar tais disfunçōes a fim de propor as intervenções específicas de enfermagem, particularmente aquelas que relacionam-se com as atividades da vida diária do indivíduo afetado.

Alguns estudos demonstram o quanto as funções cognitivas podem estar afetadas nesses pacientes.

TATE et al $^{13}$ fizeram uma análise dos aspectos neuropsicológicos em 85 pacientes que tiveram trauma de crânio e detectaram alteração em $70 \%$ deles. Os distúrbios de aprendizagem e memória foram os déficits mais freqüentemente encontrados $(56,55 \%)$.

LEVIN et $\mathrm{al}^{9}$ analisaram 127 pacientes vítimas de trauma grave listados em um Banco de Dados de coma por trauma. Verificaram que as alterações comportamentais, um ano após o trauma foram caracterizados como distúrbios de memória e lentidão no processamento de informações.

Tais resultados demonstram que a avaliação das funções cognitivas é importante e necessária, principalmente após a fase aguda do trauma.

Um paciente com TCE grave que sobrevive ao evento, em geral, é acompanhado em tratamento ambulatorial.

Nesta fase, a avaliação das funções cognitivas poderia ser feita por meio de baterias completas de teste de avaliação neurocomportamental, testes que avaliam função cognitivas específicas ou testes "screening".

Acredita-se que no âmbito do ambulatório é conveniente utilizar um método de avaliação rápido, passível de ser aplicado por profissionais sem alto grau de especialização e prolongado treinamento e que também permita uma avaliação seriada para acompanhamento da recuperação do paciente.

Neste enfoque, o teste "screening"- Mini-exame do Estado Mental (MEM) desenvolvido e validado por FOLSTEIN et al $^{6}$ tem sido bastante usado tanto em pacientes clínicos como psiquiátricos ou principalmente geriátricos 4,6,7,8,10,11,12. O MEM é um teste que concentra-se na avaliação cognitiva da função mental, testa a orientação no tempo e no espaço, a capacidade de registro de dados, a atenção e cálculo, a memória e a linguagem. O escore total máximo é de 30 sendo que valores menores ou igual a 23 têm sido apontados como indicativos de indivíduos com prejuízo cognitivo $4,11,12$.

Há também comprovação de que o MEM produz escores confiáveis os quais parecem não serem influenciados pela repetição ou aprendizagem e que, resultados consistentes são obtidos pelo mesmo ou por diferentes examinadores, em testes repetidos em intervalo de 24 horas. Resultados semelhantes foram encontrados em pacientes estáveis num intervalo médio de tempo de 28 a 31 dias ${ }^{4,6}$.

As dificuldades na orientação, atenção, memória e linguagem podem interferir na capacidade da pessoa de auto-cuidar e conseqüentemente, nas atividades de vida diária. Analisar como elas se apresentam poderia ainda contribuir para esclarecer as características definidoras do diagnóstico de enfermagem-déficit cognitivo não compensado ${ }^{1}$ ou cognição prejudicada relacionada aos aspectos do estado mental ${ }^{10}$. 
É, portanto, objetivo deste estudo analisar as disfunções cognitivas apresentadas pelos pacientes após TCE grave, por meio da utilização do MEM.

\section{MATERIAIS E MÉTODOS}

O estudo foi realizado no Ambulatório de Trauma de Crânio do Hospital das Clínicas da Faculdade de Medicina da USP (HCFMUSP)

\section{População}

Foi constituída pela totalidade dos pacientes matriculados para seguimento ambulatorial neste serviço, durante o período de agosto de 1991 a agosto de 1992. A seleção dos pacientes para matrícula é feita pelo neurocirurgião baseada em critérios de gravidade do traumatismo crânio-encefálico (TCE). Foram excluídos os pacientes com idade inferior a 12 anos e aqueles cuja data do trauma era superior a 1 ano.

\section{Métodos}

O paciente foi avaliado ao comparecer à sua primeira consulta de seguimento ambulatorial, por duas enfermeiras, autoras deste estudo. $O$ exame foi realizado, antes ou após a consulta médica, individualmente e em um dos consultórios deste serviço.

Cumpre mencionar que, os dados colhidos para este estudo faziam parte do roteiro utilizado para a primeira consulta de enfermagem. Assim, o "rapport" que deve anteceder a aplicação do teste foi estabelecido enquanto se obtinha os dados de identificação do paciente e a investigação sobre as circunstâncias do evento traumático que culminaram com o TCE. A seguir era feito o exame neurológico começando pelo MEM. Durante toda a consulta o familiar que, em geral, acompanhava o paciente, foi inserido neste contexto.

$O$ instrumento de coleta de dados foi o MEM desenvolvido e testado por FOLSTEIN et $\mathrm{al}^{6}$ e adaptado por NITRINI ${ }^{12}$ para uso no Brasil (ANEXOI). A pontuação máxima é de 30 e nos indivíduos normais, obtêm-se de 24 a 30 pontos $^{6}$. NITRINI ${ }^{12}$ estabeleceu em 4 anos a escolaridade necessária para atingir a pontuação de normalidade.

A comparação estatística entre os resultados obtidos no MEM por indivíduos com escolaridade igual ou maior que 4 anos e aqueles menores que 4 anos foi realizada pelo teste qui-quadrado $\left(x^{2}\right)$, ao nível de significância de $5 \%$. Seguiu-se a indicação de BERQUO2, utilizando-se o valor do qui-quadrado com correção de continuidade. O coeficiente de associação de Yule foi aplicado para caracterizar a magnitude e o sentido da associação. 
Consecutivamente, no período de um ano, 87 pacientes foram avaliados pelo MEM. Todos estavam conscientes e, em geral, acompanhados por um familiar.

Antes de iniciar o tratamento ambulatorial eles haviam estado internados no HCFMUSP devido trauma craniano grave e tinham como diagnóstico de lesão: contusão ou hematoma intracraniano, afundamento craniano, ferimento penetrante por arma de fogo, lesão axonal difusa ou apresentaram episódios convulsivos durante a internação ou logo após a alta hospitalar.

A distribuição dos pacientes segundo idade, sexo e causa externa pode ser vista nas Tabelas 1 e 2 .

Tabela 1. Pacientes segundo idade e sexo na primeira consulta ambulatorial pós TCE. Såo Paulo, 1991-92.

\begin{tabular}{lrrr}
\hline Idade & Masculino & Feminino & Total \\
\hline menos de 14 & - & $1(1,2)$ & $1(1,2)$ \\
\hline $15:-25$ & $28(32,2)$ & $4(4,5)$ & $32(36,7)$ \\
\hline $25:-35$ & $21(24,1)$ & $1(1,2)$ & $22(25,3)$ \\
\hline $35:-45$ & $9(10,3)$ & $1(1,2)$ & $10(11,5)$ \\
\hline $45:-55$ & $8(9,2)$ &.- & $8(9,2)$ \\
\hline 55 ou + & $12(13,8)$ & $2(2,3)$ & $14(16,1)$ \\
\hline Total & $78(89,6)$ & $9(10,4)$ & $87(100,0)$
\end{tabular}

A maioria dos pacientes eram do sexo masculino. EDNA ${ }^{5}$ encontrou predomínio masculino de $70 \%$, ou seja, um pouco menor do que o deste estudo.

Quanto a idade, houve predomínio dos mais jovens, como já era esperado. Entretanto, acima da faixa etária de 55 anos foi também encontrado $16,1 \%$ de pacientes, o que poderia ser explicado pelo tipo de causa externa que ocasionou o TCE. A idade máxima foi de 74 anos. Resultados semelhantes foram também detectados por EDNA ${ }^{5}$.

Tabela 2. Pacientes segundo causa externa e faixa etária na primeira consulta ambulatorial por TCE. Săo Paulo, 1991-82.

\begin{tabular}{|c|c|c|c|c|c|c|}
\hline \multirow[t]{3}{*}{ CAUSA EXTERNA } & \multicolumn{4}{|c|}{ Idade } & \multirow{2}{*}{\multicolumn{2}{|c|}{ TOTAL }} \\
\hline & \multicolumn{2}{|r|}{$<44$} & \multicolumn{2}{|r|}{$>45$} & & \\
\hline & $\mathbf{N}$ & $\%$ & $\mathrm{~N}$ & $\%$ & $\mathrm{~N}$ & $\%$ \\
\hline Acidentes de tránsito & 34 & $(39,1)$ & 4 & $(4,6)$ & 38 & $(43,7)$ \\
\hline Quedas & 12 & $(13,8)$ & 12 & $(13,8)$ & 24 & $(27,6)$ \\
\hline Tentativa de homicidio & 11 & $(12,6)$ & 3 & $(3,5)$ & 14 & $(16,1)$ \\
\hline Tentativa de suicidio & 1 & $(1,1)$ & 1 & $(1,1)$ & 2 & $(2,2)$ \\
\hline Outros * & 7 & $(8,1)$ & 2 & $(2,3)$ & 9 & $(10,4)$ \\
\hline Total & 65 & $(74,7)$ & 22 & $(25,3)$ & 187 & $100,0)$ \\
\hline
\end{tabular}

* Inclui-se em outros: impacto acidental contra objetos ou pessoas (2); impacto acidental causado pela queda de um objeto (2); acidentes causados por arma de fogo (1); acidente envolvendo animal montado (1); acidente causado por veículo a pedal (1); lesāo devido a queda de lugar elevado da qual se ignora se foi acidental ou intencionalmente infligida (1) e acidente causado por máquina (1). 
Os acidentes de trânsito e as quedas foram as causas externas que mais freqüentemente ocasionaram o TCE. No Brasil, naqueles que morrem devido causas externas, os acidentes de trânsito situam-se em primeiro lugar, seguido pelo homicídio ${ }^{3}$.

Analisando-se as causas externas de acordo com a idade verifica-se que os acidentes de trânsito e as tentativas de homicídio prevaleceram nos mais jovens. Já as quedas apresentam uma distribuição mais eqüitativa. De certa forma, pôde-se observar que as quedas ocorridas no domicílio estiveram mais relacionadas aos pacientes com idade mais avançadas.

Um fato que merece ser destacado é a tendência da mortalidade por causas externas, no município de São Paulo. Se naqueles que sobrevivem, o mais freqüente é o acidente de trânsito, naqueles que morrem, em primeiro lugar, situa-se o homicídio com tendência sempre crescente a partir da década de 80 e segundo lugar, o acidente de trânsito ${ }^{3}$.

Dos 87 participantes deste estudo, 7 encontravam-se afásicos inviabilizando a aplicação do MEM, portanto, este exame foi aplicado em 80 pacientes sendo seus resultados apresentados na TABELA 3 distinguindo-se indivíduos com escolaridade igual ou maior de 4 anos dos demais.

Tabela 3. Pacientes segundo pontuação do MEM na primeira consulta ambulatorial pós TCE e escolaridade em anos. São Paulo, 1991.92.

\begin{tabular}{|c|c|c|c|c|c|c|}
\hline \multirow[t]{3}{*}{ Pontuação } & \multicolumn{6}{|c|}{ Escolaridade } \\
\hline & \multicolumn{2}{|r|}{$<4$} & \multicolumn{2}{|r|}{$\geq 4$} & \multicolumn{2}{|r|}{ TOTAL } \\
\hline & $\mathbf{N}$ & $\%$ & $N$ & $\%$ & $\mathrm{~N}$ & $\%$ \\
\hline$\geq 24$ & 7 & $(8,8)$ & 45 & $(56,2)$ & 52 & $(65,0)$ \\
\hline$\leq 23$ & 19 & $(23,7)$ & 9 & $(11,3)$ & 28 & $(35,0)$ \\
\hline \multirow[t]{3}{*}{ Total } & 26 & $(32,5)$ & 54 & $(67,5)$ & 87 & $(100,0)$ \\
\hline & \multicolumn{2}{|c|}{$x^{2} c=22,1$} & & & \multicolumn{2}{|c|}{$\mathrm{X}^{2}$ crítico $5 \%=3,84$} \\
\hline & \multicolumn{2}{|c|}{$Q=-0,9$} & & & & \\
\hline
\end{tabular}

Como pode ser visto na Tabela 3 , utilizando como ponto de corte, no escore $\leq 23$ foram detectados $28(35,0 \%)$ pacientes.

Em relação ao ponto de corte, DICK et $\mathrm{al}^{4}$ estudando o MEM em pacientes com diferentes distúrbios neurológicos, verificaram que uma ótima especificidade e sensibilidade foram atingidas usando o escore de 23 ou menos como sugestivo de prejuízo cognitivo. Contudo, eles enfatizam que alguns pacientes classificados como tendo alterações cognitivas obtiveram mais que 23 pontos e alguns sem alteração, menos que 23 . Recomendam então que a interpretação dos resultados do MEM seja feita associada a outros dados clínicos.

Especificamente em pacientes com TCE grave, FRUTIGER et $\mathrm{al}^{7}$ aplicando o MEM em 91 pacientes traumatizados com 5 anos de seguimento, verificaram que $77 \%$ tenham estado mental normal e $23 \%$ estavam demenciados, principalmente em grau mínimo. 
Poder-se-ia pensar que a proporção mais elevada de pacientes com MEM inferior a $\leq 23$ que foi de $35 \%$, estivesse relacionada ao intervalo de tempo

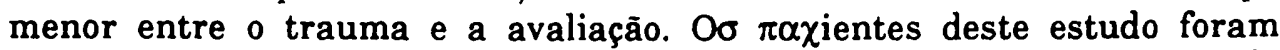
avaliados num periodo médio de 74 dias, com mínimo de 16 dias e máximo de 304 dias.

Entretanto, HOUSE et $a^{8}$ analisando pacientes no primeiro mês após acidente vascular cerebral encontraram $26 \%$ com MEM em nivel de alteração. No intervalo de 6 a 12 meses, esta proporção foi reduzida para 21\%. Assinalam que os escores baixos do MEM estiveram associados com idades mais avançadas, incapacidades físicas já existentes antes do acidente cerebral, extensão do icto e outras alterações não relacionadas a ele.

Um outro fator a ser analisado seria a escolaridade dos pacientes. Nos pacientes cuja escolaridade era inferior a 4 anos, a relação $M E M \leq 23$ foi de 2:1 quando comparando àqueles com escolaridade superior a 4 anos (Tabela 3). MURDEN et al ${ }^{11}$ estudando 358 indivíduos dos quais 100 eram brancos não demenciados, 148 negros não demenciados e 110 negros demenciados, todos com idade superior a 60 anos, concluíram que os resultados do MEM são afetados pela escolaridade mas não pela raça. Verificaram que o desempenho foi reduzido naqueles que tinham escolaridade inferior a 8 anos.

No Brasil, NITRINI ${ }^{12}$ aplicando o MEM em 362 individuos normais verificou que, apenas $4(1,5 \%)$ dos 270 indivíduos com escolaridade de 4 ou mais anos, tiveram resultado inferior a 24 pontos, enquanto que $22(24 \%)$ daqueles 92 indivíduos com escolaridade menor que 4 anos, tiveram pontuação < 24. Desta forma os resultados obtidos mostraram-se menos específicos quando o teste foi aplicado em indivíduos com escolaridade abaixo de 4 anos.

Neste estudo, observou-se, que houve uma forte associação negativa (coeficiente de Yule $=-0,9$ ) entre a obtenção da pontuação maior ou igual 24 e a escolaridade menor que 4 anos. Este resultado aponta para uma interferência da escolaridade abaixo de 4 anos nos resultados obtidos, levando indivíduos com esta escolaridade a um pior desempenho, reforçando assim a indicação do estudo de NITRINI ${ }^{12}$ que aponta como ponto de corte o valor entre 23 e 24, nos indivíduos com escolaridade de 4 anos ou mais.

Considerando a escolaridade como fator interveniente na pontuaçāo obtida no MEM, MURDEN et al ${ }^{11}$ sugerem que nos pacientes com escolaridade superior a 8 anos o ponto de corte seja mantido em 23 e 24 pontos. Recomendam que nos pacientes com escolaridade inferior a 8 anos, o ponto de corte fique em 17 ou menos pontos a fim de ser usado como indicativo de demenciação.

FOLSTEIN et $\mathrm{al}^{6}$ por sua vez, ao estandartizar o teste administrando-o em 63 idosos normais e 137 pacientes com diferentes distúrbios mentais verificaram que o escore de 20 ou menos foi encontrado somente nos pacientes com demência, delírio, esquizofrenia ou distúrbio afetivo e não nos idosos normais ou nos pacientes com diagnóstico primário de neurose ou distúrbio de personalidade. Estes autores não fazem nenhuma referência quanto a escolaridade da população estudada. 
DICK et al $^{4}$ verificando a sensibilidade e especificidade do MEM para detectar os prejuizos cognitivos, embora também não tenham estudado particularmente 0 aspecto escolaridade, observou que 4 dos 93 pacientes neurológicos com cognição intacta obtiveram escore de 23 ou menos pontos. Foi notável nesses 4 pacientes o baixo nível educacional. Para esses autores, abaixar o ponto de corte para 22 ou 21 evitaria erros de classificação, de indivíduos normais como aqueles com prejuízo de cognição, porém, reduzir o ponto de corte diminui a sensibilidade do teste, pois aumenta o número de indivíduos com prejuízos que possam ser considerados normais.

NITRINI ${ }^{12}$ considerou em seu estudo que analfabetos não podem obter mais que 28 pontos no MEM (escrita e leitura valem 2 pontos) e que portanto a especificidade poderia ser presumivelmente mantida através da redução do valor de corte nessa situação. No entanto, este mesmo autor, apresenta resultados de outros testes, que aparentemente não dependem da alfabetização e do aprendizado escolar, os quais indicam que a privação da escolaridade elementar não reduz o desempenho apenas em tarefas que incluam escrita ou leitura. Desta forma, conclui que a simples diminuição do valor de corte do MEM não o torna teste adequado para a parcela da população de baixa escolaridade.

Neste estudo, no que se refere à diminuição do ponto de corte e escolaridade, é interessante deixar registrado que dos 19 pacientes com MEM $\leq 23$ e escolaridade inferior a 4 anos, somente 8 obtiveram MEM $\leq 17$. Destes, 5 eram analfabetos, 2 tinham 2 anos de escolaridade, e um frequentou até a $3^{\mathrm{a}}$ série do primeiro grau.

Ainda abordando escolaridade, NITRINI ${ }^{12}$ comenta que indivíduos que frequentaram mais de 11 anos de ensino regular apresentaram uma média de pontos de 29,65 e que nenhum obteve menos que 27 pontos no MEM, indicando que um aumento do ponto de corte nessa população poderia aumentar a sensibilidade do teste. No presente estudo somente um dos participantes apresentava escolaridade superior a 11 anos, não fornecendo subsídios a esse respeito.

Acredita-se que a interferência do nível de escolaridade é um fator a ser considerado na aplicação do MEM. No entanto, não pode-se deixar de enfatizar, que os resultados obtidos neste exame são valores que devem ser referendados tanto nas queixas de pacientes e familiares como na própria experiência e conhecimento do profissional que realiza a avaliação.

A mudança de comportamento é um fato freqüentemente relatado tanto pelo próprio paciente como pelo familiar que o acompanha, o que sem dúvida é um forte indicador de presença de alterações. Por outro lado, o MEM também tem seu valor assegurado na avaliação da evolução do paciente, aonde a análise dos resultados têm como referencial a própria performance anterior do paciente.

Além de detectar o MEM com resultados indicativos de alteração cognitiva, considerou-se importante verificar quais seriam os itens que encontramse alterados. 
Assim, mantendo-se o ponto de corte em $\leq 23$ foi feita uma análise dos itens do MEM que foram detectados como alterados. Definiu-se como item alterado, toda vez que o paciente não obtinha a pontuação máxima no mesmo.

Tabela 4. itens alterados no MEM nos pacientes com pontuação / 23 segundo escolaridade. São Paulo, 1991-92.

\begin{tabular}{lrrrrr}
\hline ALTERAÇÃO DO MEM & \multicolumn{1}{c}{ Escolaridade } \\
\hline & SIM & NÄO & SIM & TOTAL \\
\hline Orientação & 16 & 3 & 9 & - & NÃO \\
\hline Registro de Dados & 3 & 16 & - & 9 & 28 \\
\hline Atenção e Cálculo & 19 & - & 8 & 1 & 28 \\
\hline Memória de fixação & 14 & 5 & 6 & 3 & 28 \\
\hline Linguagem & 18 & 1 & 7 & 2 & 28
\end{tabular}

Como pode ser visto na Tabela 4, o único item que praticamente não esteve alterado foi o de "registro de dados" ou seja, aquele que testa a memória imediata. A proporção de alterados nos demais itens foi sempre alta e predominou tanto naqueles com escolaridade inferior como superior a 4 anos. Mesmo no item "linguagem" onde os indivíduos com escolaridade menor que 4 anos têm maior probabilidade de ter um desempenho mais deficiente, as alterações apareceram de forma semelhante.

A apresentação de alterações distribuídas em vários itens do domínio cognitivo poderia ser atribuída a localização diversificada do foco de lesão dos pacientes, porém, observando-se a característica desta distribuição que aponta para vários itens alterados em um mesmo paciente, evidencia-se que nos traumatismos crânio-encefálicos graves os danos são múltiplos.

\section{CONCLUSÕES}

- Na primeira consulta de enfermagem, $28(35,0 \%)$ dos pacientes em tratamento ambulatorial apresentaram disfunção cognitiva detectada por $\mathrm{MEM} \leq 23$.

- O MEM $\leq 23$ foi mais freqüente nos pacientes com escolaridade inferior a 4 anos em relação àqueles superiores a 4 anos. Esta relação foi de $2: 1$.

- Houve uma forte associação negativa (coeficiente de Yule= -0,9) entre a obtenção de pontuação maior ou igual a 24 e a escolaridade menor de 4 anos.

-- Quase todos os itens do MEM com exceção do registro de dados apresentaram-se alterados naqueles cujo escore foi $\leq 23$ pontos, tanto naqueles cuja escolaridade inferior como superior a 4 anos. 
KOIZUMI, M.S. et al. Mini-Menthal state exam - indicator cognitive alteration after severe traumatic brain injury. Rev.Esc.Enf.USP, v.28,n.3, p.281-92, dec. 1994.

The goal of this study is to analyse the mental state and cognitive alterations presented by outpatients starting ambulatory care after severe traumatic brain injury. During 1 year, consecutively, 87 patients were attended in first medical an nursing consultation. Mini-mental State exam (MMS) were applied to 80 of them. The average number of days between the injury and MMS application was 74 days. We used 23 score or less as a cutoff point. $35 \%$ of the patients showed less than the 23 score. When we compared the patient with 23 or less MMS score, the groups of patients whose studies were lower than or up to the $4^{\text {th }}$ grade of elementary school, the relationship was found to be 2:1. The statistical testes (Chi-square and Yule) showed a strong negative association between the score equal or higher than 24 and an education worse than the $4^{\text {th }}$ grade $(Q=-0,9)$. The item disturbed less even in the 23 or less MMS score patients, was the registration. The other itens: orientation, attention and calculation, recall and language were disturbed in all patients in different proportions. In regard to nursing interventions derived from cognitive alteration nursing diagnosis, we believe that besides special attention for the patient it is necessary to notify the family about the patient's disturbances and how to care of them during convalescence period.

UNITERMS: Cognitive disfunction. Mental State. Head injury.

\section{REFERÊNCIAS BIBLIOGRÁFICAS}

1. AMERICAN NURSES'S ASSOCIATION Neuroscience nursing practice: process and outcome criteria for selected diagnoses. Kansas City, 1985 (publ. MS-13)

2. BERQUO, E.S. et al. Bioestatística. São Paulo, EPU, 1980.

3. BRASIL. Ministério da Saúde. Secretaria Nacional de Açōes Básicas de Saúde. Subsistema de Informação sobre mortalidade. Estatística de mortalidade: Brasil, 1985. Brasilia, Centro de Documentação do Ministério da Saúde, 1988 (Série G: Estatística e informação de Saúde, 16).

4. DICK, J.P.R. et al. Mini-mental state examination in neurological patients. J.Neurol, v.17, p.496-9, 1984.

5. EDNA, T.H. Head injuries admitted to hospital-epidemiology, risk factors and long term outcome. J.Oslo City Hosp., v.37, n.9, p.101-6, 1987.

6. FOLSTEIN, M.F. et al. Mini-mental: a pratical method for grading the cognitive state of patients for the clinican. J.Psychiatr.Res., v.12, n.3, p.189-98, 1975.

7. FRUTIGER, A. et al. Five years follow-up of severely injured ICU patients. J.Trauma,v.31, n.9, p. 1216-25, 1991.

8. HOUSE, A. et al. The relationship between intellectual impairment and mood disorder in first year after stroke. Psychol Med., v.20, n.4, p. 805-14, 1990.

9. LEVIN, H.S. et al. Neurobehavioral outcome 1 year after severe head injury: experience of the traumatic coma date bank. J. Neurosurg., v.73, n.5, p. 699-709, 1990.

10.MITCHELL, P.H. et al. AANN'S Neuroscience Nursing: phenomena and practice. Norwalk, Appleton \& Lange, 1988. 
11.MURDEN, R.A. et al. Mini-mental state exam scores vary with education in blacks and whites. J.Am.Genatr.Soc., v.39, n.2, p. 149-55, 1991.

12.NITRINI, R. et al. Condutas em neurologia - 1991. Sảo Paulo, Clínica Neurológica do HC/FMUSP, 1991. Cap. 37, p.145-51: Demència-avaliação e conduta.

13.TATE, R.L. et al. Patterns of neuropsychological impairment after severe blunt head injury. J.Nerv.Ment.Dis., v.179, n.3, p. 117-26, 1991. 


\section{ANEXO I \\ MINI-EXAME DO ESTADO MENTAL}

(Folstein, Folstein e MCHugh - 1975)

Nome:

Idade:

Número de anos que frequentou a escola:

ORIENTAÇÃo (10 PONTOS)

1. Dia da semana

2. Dia do mès

3. Mês

4. Ano

5. Hora aproximada

6. Local onde se encontra

7. Endereço (ou como chegou ao local do exame)

8. Andar ou setor

9. Cidade

10. Estado

\section{REGISTRO DE DADOS (3 PONTOS)}

- três palavras (vaso, carro, janela)

\section{ATENÇÃO E CÁLCULO (5 PONTOS)}

- 100-7 sucessivos ou soletrar "mundo" ao inverso

\section{MEMÓRIA DE FIXAÇÃO (3 PONTOS)}

- recordar as palavras do item de dados

\section{LINGUAGEM (9 PONTOS)}

- nomear uma caneta e um relógio ( 2 pontos)

- repetir "nem aqui, nem ali. nem lá"

- obedecer a ordem: "pegue este papel com sua mão direita, dobre-o ao meio e o coloque no chāo" ( 3 pontos)

- ler e obedecer: "feche os olhos"

- escrever uma frase

- copiar um desenho

\section{TOTAL}




\section{ANEXO I \\ INSTRUÇÃO PARA REALIZAÇÃO DO MINI-EXAME DO ESTADO MENTAL}

\section{ORIENTAÇĀO}

Pergunte qual a data de hoje. Em seguida pergunte as partes omitidas. Aproximação de $+/-1$ hora é aceita como correta.

Endereço pode ser substituído por uma explicação razoável de como chegou ao local.

\section{RETENÇÃo}

Pergunte ao paciente se você pode testar sua memória. Diga o nome de três objetos, claramente, cerca de 1 segundo para cada palavra. A pontuação é dada pela primeira repetição. Continue repetindo as palavras até 6 tentativas.

\section{ATENÇÃo E CÁlCULO}

Peça para retirar 7 de 100 , sucessivamente. Corrija quando o resultado estiver errado e peça para continuar subtraindo. Pare após 5 resultados (65). Se o paciente näo for capaz, peça-lhe para soletrar a palavra "mundo" de trás para diante.

Atribua um ponto para cada acerto, valorizando apenas o teste em que o desempenho tenha sido melhor.

\section{MEMÓRIA}

Peça ao paciente para repetir os nomes de objetos que você lhe havia pedido para lembrar-se há pouco.

\section{LINGUAGEM}

Mostre um relógio de pulso e pergunte qual o nome. Repita com uma caneta. Peça ao paciente para repetir a frase "nem aqui, nem ali, nem lá" depois de você (uma única tentativa).

Coloque uma folha de papel em branco sobre a mesa e dê a ordem para pegála com a mão direita, etc. Anote um ponto para cada parte corretamente executada.

Peça para escrever uma frase para o médico. Deve ter sujeito e um verbo e ter sentido; não é necessário estar graficamente correta. Ou em um papel em branco, desenhe dois pentágonos interseccionados, com cerca de $2,5 \mathrm{~cm}$ de lado e peça para copiar exatamente como estāo desenhados. Todos os 10 ângulos e a intersecção devem ser desenhados. 